for 48 hours tracheal intubation is a justifiable alternative to tracheostomy.

Tonkin and Harrison also clarify the factors responsible for laryngeal damage after intubation. They suggest that the important ones are trauma to the mucous membrane during intubation, female sex of the patient, and the presence of pre-existing laryngeal oedema caused by overinfusion. Duration of intubation beyond 48 hours and nature of the material of which the tube or the lubricant is composed are factors whose influence is not yet clear. They are in the process of being studied prospectively.

Prolonged endotracheal intubation also appears to be particularly indicated for infants and small children, in whom tracheostomy is associated with a high incidence of complications. $^{2}$ For infants nasal tubes are mostly used, and are generally made of polyvinyl chloride. One in particular ${ }^{5}$ has a crosspiece for connexion to the ventilator or other apparatus. Nevertheless, subglottic stenosis has been reported $^{5-7}$ after prolonged nasal endotracheal intubation in infants and small children. This is a serious matter, because as well as endangering life by causing respiratory obstruction stenosis is difficult to treat surgically. In an attempt to reduce the risk of this particular complication it is suggested ${ }^{7}$ that the tube should be small and that it should be lubricated with $1 \%$ hydrocortisone ointment in order to minimize any local inflammatory response. ${ }^{\circ}$

Accumulating experience of prolonged endotracheal intubation suggests that it can be a satisfactory alternative to tracheostomy, particularly in two types of patient: those with respiratory difficulty which is not likely to last longer than 48 hours, and small children and infants even if a longer period of artificial ventilation is needed.

\section{Ulcerative Colitis and Cancer}

Ulcerative colitis is a diffuse ulcerative condition of unknown aetiology affecting the mucous membrane of part or the whole of the large bowel. In the early stages there is an acute inflammatory change in the mucosa, and the crypts of Lieberkühn are distended with pus cells, inflammatory exudate, and mucus. ${ }^{1}$ These " crypt abscesses" are typical of ulcerative colitis, and as the crypt walls disintegrate and the overlying mucosa undergoes necrosis areas of ulceration are produced, which extend to the submucosa. In the most fulminating cases the inflammation spreads through the muscle coat to the peritoneal surface, and the bowel may undergo perforation. Usually the process remains superficial, and the destroyed mucosa and submucosa are replaced by granulation tissue, which when it matures to fibrous tissue causes shortening and stenosis of the bowel. During the phases of ulceration and healing portions of the mucous membrane are often elevated into polypoid excrescences. Some of these are merely ragged remnants of mucosa surviving amid extensive areas of ulceration. Others consist of a core of exuberant granulation tissue covered by a layer of epithelium. These mucosal projections are called pseudopolyps, because there is no increase in epithelial growth. They are not particularly prone to develop malignant change.

In the later stages of the disease the epithelium may undergo changes that culminate in cancer. True polypoid overgrowth of the mucosa is an early precancerous lesion, and it is sometimes seen scattered over much of the bowel. ${ }^{2}$ Probably between 3 and $5 \%$ of all cases of ulcerative colitis are complicated by carcinoma of the large bowel, ${ }^{3}$ while the risk of this complication is between five and ten times greater than in people without the disease. ${ }^{4}$ It is generally held that the prognosis of cancer in ulcerative colitis is worse than in sporadically occurring colonic cancer. ${ }^{5}$ The tumours are often multiple, and many of them are poorly differentiated or mucus secreting. ${ }^{6}$ Prophylactic proctocolectomy would certainly obviate the hazard of patients developing cancer, but it would lead to many patients who are not destined to develop cancer being subjected to a major operative procedure.

To define those groups of patients with ulcerative colitis who have a serious risk of developing carcinoma, J. M. Hinton has investigated a series of 32 patients with both lesions who were treated at St. Mark's Hospital in London. ${ }^{7}$ He found that when the colitis was confined to the rectum and sigmoid colon the risk of cancer was no greater than in the general population. When the disease affected the whole colon, on the other hand, the risk was greatest. Other important. factors were the duration of the colitis and the age of onset. Cancer was more likely to occur in those patients who were younger than 25 years when the colitis began, and it became an increasingly serious risk as the duration exceeded 10 years. Though the risk was very low for the first 10 years, it rose after this age-until by 25 years the cumulative risk was $42 \% .^{6}$ In Hinton's series of cases seven patients had more than one cancer present in the colon, and the transverse colon was affected much more frequently than it is in sporadically occurring carcinoma.

Other types of malignancy have also been reported as complications of ulcerative colitis. Primary lymphosarcoma of the large bowel-a rare condition-has been described in association with long-standing colitis on seven occasions. ${ }^{8}$ In another large series of cases of colitis a significantly increased incidence of carcinoma of the alimentary tract apart from the large bowel was found, and it was suggested that a widespread disturbance of the digestive system occurred in this disease. ${ }^{3}$ Another neoplasm that has been reported in association with ulcerative colitis is carcinoma of the bile ducts. J. G. Rankin and his colleagues have recently reported three cases of this uncommon tumour in a series of 441 patients with colitis. ${ }^{9}$ In every instance the colitis had been present for over 10 years before the carcinoma became clinically evident, and two of the patients died at the age of 22 years. Though pericholangitis is known to occur quite frequently in colitis, there was little to incriminate antecedent biliary disease in these three patients.

The relation between carcinoma of the bowel and ulcerative colitis still has many unexplained features. There appears to be no similar association between cancer and Crohn's disease of the large bowel-which suggests that the epithelial changes in colitis are of a different character. Despite the admirable epidemiological surveys designed to define the imminence of carcinoma, the lives of patients with ulcerative colitis will continue to be in jeopardy until precancerous lesions of the colon can be diagnosed with greater certainty.

\footnotetext{
Lumb, G., and Protheroe, R. H. B., Lancet, 1955, 2, 1208.

Dawson, I. P., and Pryse-Davies, J. Brit. F. Surg., 1959, 47, 113.

Edwards, F. C., and Truelove, S. C., Gut, 1964, 5, 1.

Goldgraber, M. B., and Kirsner, J. B., Cancer (Baltimore), 1964, 17 657.

Slaney, G., and Brooke, B. N., Lancet, 1959, 2, 694.

6 Morson, B. C., Gut, 1966, 7, 425.

Hinton, J. M., ibid., 1966, 7, 427.

Cornes, J. S., Smith, J. C., and Southwood, W. F. W., Brit. F. Surg., 1961, 49, 50.

- Rankin, J. G., Skyring, A. P., and Goulston, S. J. M., Gut, 1966, 7, 433 .
} 